\title{
Paraganglioma of Urinary Bladder in a Pediatric Patient
}

\author{
Shoaib Muhammad ${ }^{1}$, Amman Yousaf ${ }^{2,3}$, Arif Qayyum ${ }^{1}$, Rabia Nazim ${ }^{4}$, Muhammad Taqi ${ }^{5}$ \\ 1. Department of Urology, Ghulab Devi Hospital, Al-Aleem Medical College, Lahore, PAK 2. Department of Radiology, \\ Hamad General Hospital, Doha, QAT 3. Department of Radiology, Services Hospital Lahore, Lahore, PAK 4. Department \\ of Community Medicine, King Edward Medical University, Lahore, PAK 5. Department of Anaesthesiology, Ghulab Devi \\ Hospital, Al-Aleem Medical College, Lahore, PAK
}

Corresponding author: Amman Yousaf, ayousafmd2@gmail.com

\begin{abstract}
Paragangliomas arise from neural cells and are found in different anatomical locations in the body. Paragangliomas in the adrenal glands are called pheochromocytoma, while the others are known as extraadrenal paragangliomas. They are usually benign and are extremely rare in children. We present a case of a 13-year-old female patient who presented with complaints of hematuria for one year and left lower lumbar pain. Imaging investigations depicted a urinary bladder mass that was causing a mass effect at the left ureteric opening and backpressure changes in the left kidney. The patient underwent transurethral resection of bladder mass, and the histopathology confirmed the presence of paraganglioma. Though the paragangliomas of the urinary bladder are extremely rare in the pediatric age group, we suggest keeping paragangliomas on differentials when investigating a patient with bladder mass.
\end{abstract}

Review began 03/14/2021 Review ended 03/16/2021 Published 03/18/2021

๑) Copyright 2021

Muhammad et al. This is an open access article distributed under the terms of the Creative Commons Attribution License CC-BY 4.0., which permits unrestricted use, distribution, and reproduction in any medium, provided the original author and source are credited.
Categories: Pediatric Surgery, Radiology, Urology

Keywords: extra-adrenal paraganglioma, urinary bladder ca, pediatric

\section{Introduction}

Pheochromocytoma and paraganglioma are neural cell origin tumors [1]. Being histologically the same, they are differentiated based on their anatomic location, with pheochromocytoma being an adrenal gland tumor and paraganglioma being an extra-adrenal tumor. Compared to pheochromocytoma (80-85\%),

paragangliomas are extremely uncommon (15-20\%) [1]. These tumors are usually diagnosed at the ages of 30 to 50 years, and diagnosis in children is extremely rare (10\%). Most tumors are benign; however, infiltration and metastasis go in the favor of malignancy [2].

Paragangliomas in the urinary bladder being a rare entity has a prevalence of around $0.06 \%$ of all tumors, and those with malignant potential advocated by metastasis are extremely rare [3,4]. The first case of paraganglioma of the urinary bladder was reported in 1953, and since then only a few hundred cases have been reported [4]. We present a case of urinary bladder paraganglioma in a pediatric age group patient who was diagnosed during the workup for hypertension and lumbar pain. We suggest keeping paraganglioma in the differential diagnosis of the urinary bladder mass especially those who present with hypertension.

\section{Case Presentation}

A 13-year-old girl presented to the emergency department with a history of hematuria for the last one year associated with on and off left lumbar pain. Her family history was insignificant for any malignancy of the genitourinary tract. Her pulse was 94 beats/minute, blood pressure was 150/90 $\mathrm{mmHg}$, and respiratory rate was 17 breaths/minute. Here general physical and abdominal examination was unremarkable.

Ultrasound (USG) of kidneys and urinary bladder was performed with a full bladder, and an irregular mass was appreciated in the left posterior wall of the urinary bladder with significant left-sided hydronephrosis (Figure 1). 


\section{Cureus}

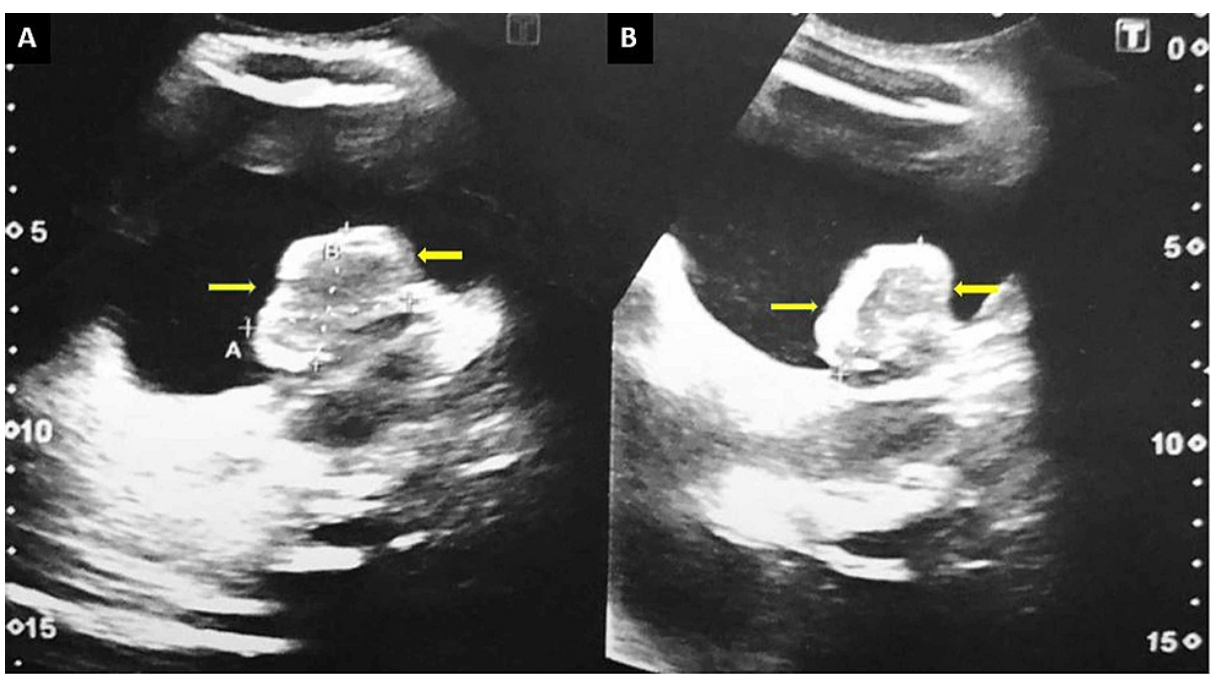

FIGURE 1: Ultrasound of the urinary bladder showing a large lobulated heterogeneously hypoechoic mass originating from the posterior wall.

Contrast-enhanced computed tomography (CT) confirmed a large urinary bladder mass in the left margin of the urinary bladder base. It had heterogeneously enhancing soft tissue density and was abutting the anterior perirectal wall and the adjacent structures. Multiple enlarged lymph nodes were seen in the left pelvic area (Figure 2).
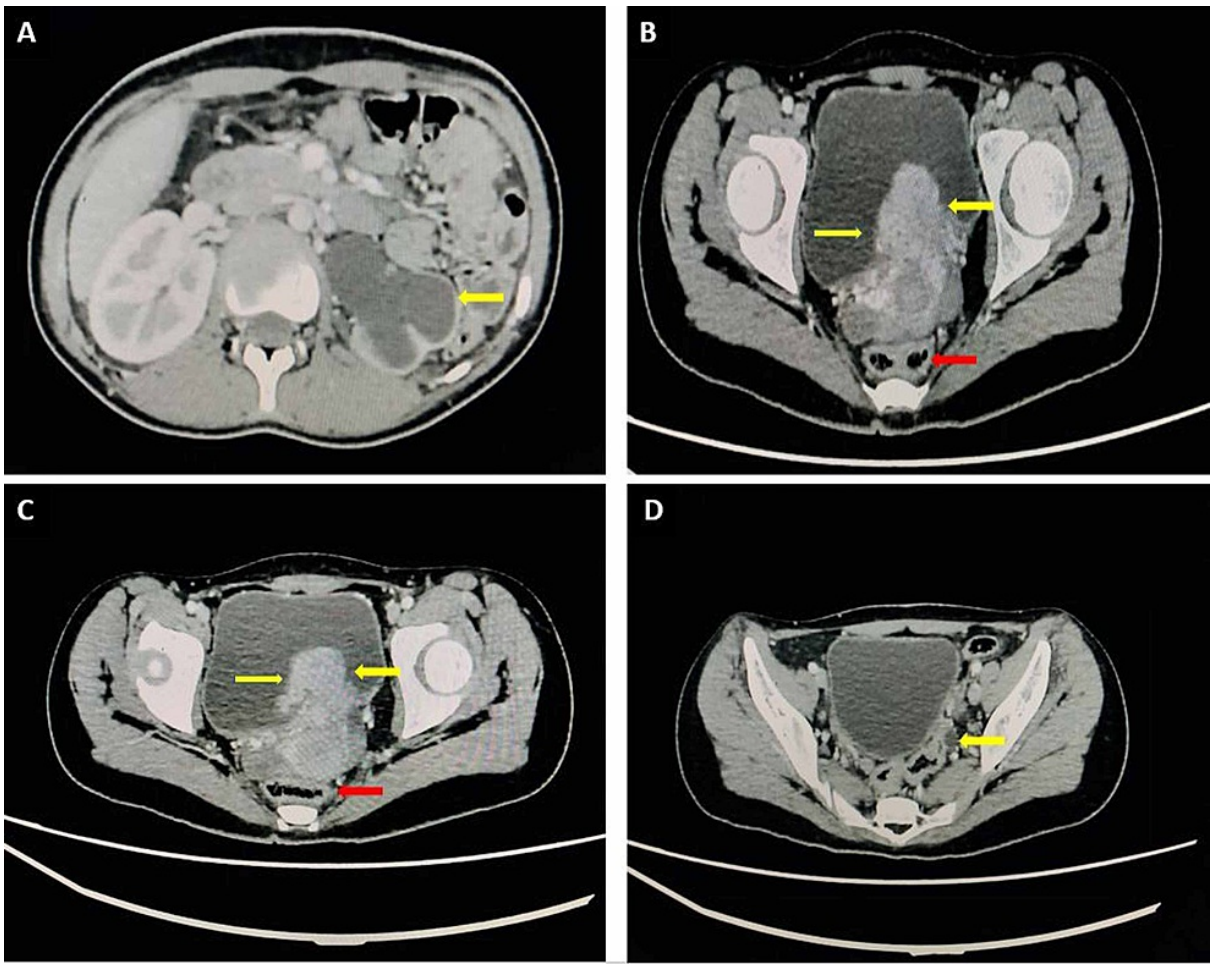

FIGURE 2: Contrast-enhanced computed tomography (CT) of the abdomen and pelvis

(A) Axial selected section at the kidneys level, demonstrating left-sided dilated pelvicalyceal system with thinning of the cortex (hydronephrosis). (B \& C) Selected axial images demonstrating a large heterogeneously enhancing mass lesion (yellow arrows) arising from the left posterior wall of the urinary bladder with no obvious rectal (red arrows) infiltration. (D) Dilated distal left ureter (arrow).

The multidisciplinary team decided to proceed with transurethral cystoscopic resection of the mass with special preoperative and intra-operative preparation (phenoxybenzamine and propranolol) to prevent any 
hypertensive crisis. Surgery and postoperative recovery went uneventful. Histopathology of the resected specimen revealed a focus of tumor cells within the bladder mucosa. The tumor cells were arranged in nests and trabeculae. Individual cells were round to oval with abundant cytoplasm and atypical nuclei. The immunohistochemistry was significant for chromogranin, synaptophysin, and S100 positivity (Figure 3).
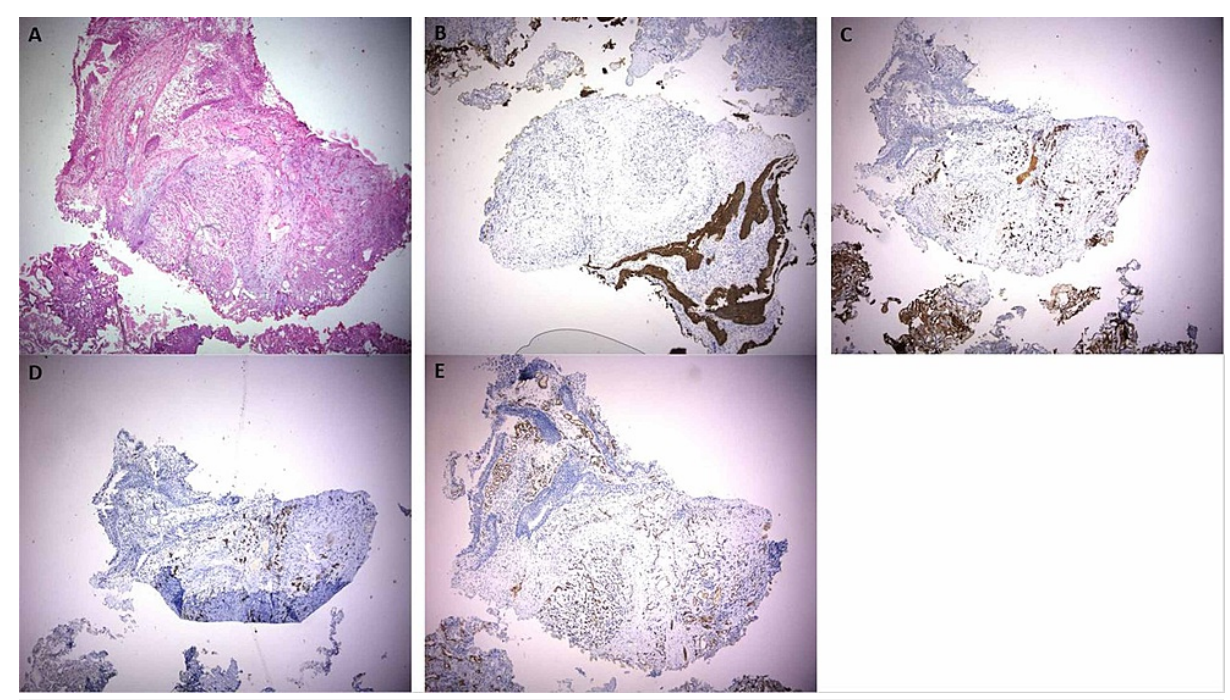

\section{FIGURE 3: Histopathological features of the resected urinary bladder mass}

Tumor cells in the urinary bladder mucosa arranged in nests and trabeculae. Individual cells are round to oval with eosinophilic cytoplasm and atypical nuclei (A). Tumor cells are negative for cytokeratin (B), whereas they are positive for chromogranin (C), synaptophysin (D), and S-100 (E).

A diagnosis of urinary bladder paraganglioma was made. The patient was discharged on the third postoperative day and is living a routine healthy life to date.

\section{Discussion}

Paragangliomas originating in the urinary bladder are extremely uncommon tumors that grow from chromaffin tissue of the sympathetic nervous system related to the urinary bladder. These growths can be active (producing secretions) or inactive (not producing any secretions) [3]. The paragangliomas can present with the clinical picture of excessive production of catecholamine (hypertension, tremors, diaphoresis, headache, and loss of consciousness after passing urine). The first case of urinary bladder paragangliomas was reported by Zimmerman et al. in 1953 [4].

Paragangliomas occurring in the urinary bladder make $0.05 \%$ of all tumors. In the genitourinary system, the urinary bladder is the most frequent site for the occurrence of paragangliomas (79.2\%), and the second most common site is the urethra (12.7\%) followed by the pelvis (4.9\%) and ureter (3.2\%). Female predominance has been reported in the literature with a female-to-male ratio of 3:1, and they usually present between 30 and 50 years of age $[5,6]$. Most tumors occurring outside adrenal glands are found in the abdomen. Around $85 \%$ of them present under the diaphragm on the side of the sympathetic chain on the site of aortic division or the beginning of the inferior mesenteric artery (from the organ of Zuckerkandl) [7]. The least frequent site of occurrence is the urinary bladder [8].

Due to the absence of typical features, these are sometimes misdiagnosed as urothelial cancers. Around 10\% of the paragangliomas in the urinary bladder are malignant [4]. Gross or histological features are not enough to differentiate between benign and malignant paragangliomas. Local infiltration of the surrounding organs, lymph nodes involvement, and metastasis to the remote organs are the characteristic features of malignant paragangliomas [8-10].

The paragangliomas of the urinary bladder usually have a lobulated macroscopic appearance. On microscopy, the tumor cells are arranged in trabeculae and nests making the zellballen pattern. Cells have acidophilic granular cytoplasm with oval-shaped nuclei [10]. Although paragangliomas occur in various locations, they usually show almost similar imaging features. CT demonstrates the paraganglioma as a heterogeneous soft tissue mass with a homogeneous or heterogeneous contrast enhancement followed by slow washout owing to a high capillary network [11]. These features were consistent with the findings in our case. On MRI, the lesions tend to be hypointense or isointense on T1 and hyperintense on T2 with interspersed areas of signal voids (salt-and-pepper appearance), with heterogeneous contrast enhancement. 
Metaiodobenzylguanidine scintigram (MIBG) has a crucial role in differentiating between functional and non-functional paragangliomas [10]. Surgical resection is the treatment of choice with adequate pre- and postoperative preparation to avoid the hypertensive crisis. Radical cystectomy, partial cystectomy, and transurethral resection of the tumor are the available surgical options [10,12-14]. In our case, transurethral resection was performed as the tumor was a single focus, with no infiltration of the surrounding structures and no distal metastasis.

\section{Conclusions}

Paragangliomas can occur in a variety of anatomical locations.. The imaging features of paragangliomas of different locations are almost identical, and local or distal metastasis differentiates between benign and malignant lesions. We suggest keeping paraganglioma in the differentials of a solid urinary bladder mass lesion in pediatric age group patients.

\section{Additional Information \\ Disclosures}

Human subjects: Consent was obtained or waived by all participants in this study. Conflicts of interest: In compliance with the ICMJE uniform disclosure form, all authors declare the following: Payment/services info: All authors have declared that no financial support was received from any organization for the submitted work. Financial relationships: All authors have declared that they have no financial relationships at present or within the previous three years with any organizations that might have an interest in the submitted work. Other relationships: All authors have declared that there are no other relationships or activities that could appear to have influenced the submitted work.

\section{References}

1. Zhou M, Epstein JI, Young RH: Paraganglioma of the urinary bladder: a lesion that may be misdiagnosed as urothelial carcinoma in transurethral resection specimens. Am J Surg Pathol. 2004, 28:94-100. 10.1097/00000478-200401000-00011

2. Erickson D, Kudva YC, Ebersold MJ, Thompson GB, Grant CS, van Heerden JA, Young WF Jr: Benign paragangliomas: clinical presentation and treatment outcomes in 236 patients. J Clin Endocrinol Metab. 2001, 86:5210-5216. 10.1210/jcem.86.11.8034

3. Sheps SG, Jiang NS, Klee GG, van Heerden JA: Recent developments in the diagnosis and treatment of pheochromocytoma. Mayo Clin Proc. 1990, 65:88-95. 10.1016/s0025-6196(12)62113-2

4. Zimmerman IJ, Biron RE, Macmahon HE: Pheochromocytoma of the urinary bladder . NEJM. 1953, 249:25-26. 10.1056/nejm195307022490106

5. Hanji AM, Rohan VS, Patel JJ, Tankshali RA: Pheochromocytoma of the urinary bladder: a rare cause of severe hypertension. Saudi J Kidney Dis Transpl. 2012, 23:813-816. 10.4103/1319-2442.98167

6. Dahm P, Gschwend JE: Malignant non-urothelial neoplasm of the urinary bladder: a review . Eur Urol. 2003, 44:672-681. 10.1016/s0302-2838(03)00416-0

7. Lam AK: Update on adrenal tumours in 2017 World Health Organization (WHO) of endocrine tumours . Endocr Pathol. 2017, 28:213-227. 10.1007/s12022-017-9484-5

8. Yadav R, Das AK, Kumar R: Malignant non-functional paraganglioma of the bladder presenting with azotemia. Int Urol Nephrol. 2007, 39:449-451. 10.1007/s11255-006-9017-5

9. Deng JH, Li HZ, Zhang YS, Liu GH: Functional paragangliomas of the urinary bladder: a report of 9 cases . Chin J Cancer. 2010, 29:729-734. 10.5732/cjc.009.10703

10. Tsai CC, Wu WJ, Chueh KS, et al.: Paraganglioma of the urinary bladder first presented by bladder bloody tamponade: two case reports and review of the literatures. Kaohsiung J Med Sci. 2011, 27:108-113. 10.1016/j.kjms.2010.05.005

11. Lee KY, Oh YW, Noh HJ, et al.: Extraadrenal paragangliomas of the body: imaging features . AJR Am J Roentgenol. 2006, 187:492-504. 10.2214/AJR.05.0370

12. Quist EE, Javadzadeh BM, Johannesen E, Johansson SL, Lele SM, Kozel JA: Malignant paraganglioma of the bladder: a case report and review of the literature. Pathol Res Pract. 2015, 211:183-188. 10.1016/j.prp.2014.10.009

13. Al-Zahrani AA: Recurrent urinary bladder paraganglioma . Adv Urol. 2010, 2010:912125. $10.1155 / 2010 / 912125$

14. Wald O, Shapira OM, Murar A, Izhar U: Paraganglioma of the mediastinum: challenges in diagnosis and surgical management. J Cardiothorac Surg. 2010, 5:19. 10.1186/1749-8090-5-19 